\title{
BMJ Open Proactive healthcare for frail elderly persons: study protocol for a prospective controlled primary care intervention in Sweden
}

\author{
Jan Marcusson, ${ }^{\oplus 1}$ Magnus Nord, ${ }^{2}$ Maria M Johansson, ${ }^{1}$ Jenny Alwin, ${ }^{3}$ \\ Lars-Åke Levin, ${ }^{3}$ Petra Dannapfel, ${ }^{1}$ Kristin Thomas, ${ }^{4}$ Bonnie Poksinska, ${ }^{5}$ \\ Annette Sverker, ${ }^{6}$ Anna Olaison, ${ }^{7}$ Elisabet Cedersund, ${ }^{8}$ Susanne Kelfve, ${ }^{7}$ \\ Andreas Motel-Klingebiel, ${ }^{7}$ Ingrid Hellström, ${ }^{9}$ Agneta Kullberg, ${ }^{7}$ Ylva Böttiger, ${ }^{10}$ \\ Huan-Ji Dong, ${ }^{4}$ Anneli Peolsson, ${ }^{11}$ Malin Wass, ${ }^{12}$ Johan Lyth, ${ }^{12}$ \\ Agneta Andersson ${ }^{12}$
}

To cite: Marcusson J, Nord M, Johansson MM, et al. Proactive healthcare for frail elderly persons: study protocol for a prospective controlled primary care intervention in Sweden. BMJ Open 2019;9:e027847. doi:10.1136/ bmjopen-2018-027847

- Prepublication history for this paper is available online. To view these files, please visit the journal online (http://dx.doi org/10.1136/bmjopen-2018027847).

Received 10 November 2018 Revised 26 February 2019 Accepted 5 April 2019
Check for updates

(C) Author(s) (or their employer(s)) 2019. Re-use permitted under CC BY-NC. No commercial re-use. See rights and permissions. Published by BMJ.

For numbered affiliations see end of article.

Correspondence to Professor Jan Marcusson; jan.marcusson@liu.se

\section{ABSTRACT}

Introduction The provision of healthcare services is not dedicated to promoting maintenance of function and does not target frail older persons at high risk of the main causes of morbidity and mortality. The aim of this study is to evaluate the effects of a proactive medical and social intervention in comparison with conventional care on a group of persons aged 75 and older selected by statistical prediction.

Methods and analysis In a pragmatic multicentre primary care setting $(n=1600)$, a prediction model to find elderly $(75+)$ persons at high risk of complex medical care or hospitalisation is used, followed by proactive medical and social care, in comparison with usual care. The study started in April 2017 with a run-in period until December 2017, followed by a 2-year continued intervention phase that will continue until the end of December 2019. The intervention includes several tools (multiprofessional team for rehabilitation, social support, medical care home visits and telephone support). Primary outcome measures are healthcare cost, number of hospital care episodes, hospital care days and mortality. Secondary outcome measures are number of outpatient visits, cost of social care and informal care, number of prescribed drugs, health-related quality of life, cost-effectiveness, sense of security, functional status and ability. We also study the care of elderly persons in a broader sense, by covering the perspectives of the patients, the professional staff and the management, and on a political level, by using semistructured interviews, qualitative methods and a questionnaire.

Ethics and dissemination Approved by the regional ethical review board in Linköping (Dnr 2016/347-31). The results will be presented in scientific journals and scientific meetings during 2019-2022 and are planned to be used for the development of future care models.

Trial registration number NCT03180606.

\section{INTRODUCTION}

The healthcare situation of the elderly is a challenge for healthcare systems in many

\section{Strengths and limitations of this study}

- This study is a pragmatic clinical trial on proactive healthcare for people 75 years and older in primary care, meaning that it has a close connection with clinical reality, which will enhance any future implementation.

- The case-finding method is a statistical prediction model that allows the 'screening' of large numbers of patients.

- The developed clinical evaluation and management model integrates primary care with community care and social services.

- The project also focuses on the perspectives of the patients, the professionals in the healthcare system and the governance mechanisms, which may explain the perceived shortcomings of today's healthcare for the elderly.

- A fairly long run-in period due to clinical realities and organisational inertia in the healthcare system, as well as a long intervention period of 2 years, is a clinical necessity, but this increases the risk of non-controlled influences on the project.

countries, and healthcare providers struggle to meet the needs of a growing number of older people. ${ }^{1}$ In Sweden, the largest consumers of medical services $(60 \%)$ are persons 80 years and older (15\% of the population), a group that is predicted to increase by $50 \%$ over the next 15 years. Several studies report that a majority of the aged population is satisfied with their health, ${ }^{2}$ manage life at home and consider themselves healthy. ${ }^{34}$ Only a minority of the aged population is in need of hospital care. In most cases, the healthcare system does not distinguish between different groups among the heterogeneous old-age population; instead, both hospital and 
primary care are organised using a passive and reactive (acting when symptoms or problems occur) approach. There have been many attempts to define and measure frailty among the elderly in order to detect persons with significant care needs (see, eg, Edmans et a $\bar{p}$ ). However, 'frailty' is difficult to define as a medical condition, and there is no consensus on the operational definition of the concept. ${ }^{6}$ Three major frailty models have been suggested: physical frailty model, deficit accumulation model of frailty and the biopsychosocial or multidimensional model. ${ }^{7}$ Furthermore, evaluation using clinical instruments requires trained staff for each individual evaluation, which is not easily applied within a broader clinical context that lacks a primary geriatric perspective (eg, primary care and acute ward disciplines).

The current healthcare system in many countries is not designed to identify individuals with healthcare needs or to direct care resources towards those with the greatest need for care prior to hospitalisation. Since the use of 'frailty scales' involves merely a fraction of the flow of hospitalised elderly, statistical prediction models have been proposed as an effective means of evaluating larger target groups to enable resource-limited interventions for those with the greatest needs. ${ }^{8}$ However, the clinical use of prediction in routine clinical primary care of the elderly remains to be clarified. Proactive interventions provided to the elderly within a certain age range, and/ or with multimorbidity but with low predictive value for hospitalisation, may direct healthcare resources towards groups that are not in most need of them. Likewise, interventions for small, specific groups (eg, newly hospitalised, specific medical diagnoses or patients above a certain frailty index score) will neglect large groups of elderly in need of healthcare or miss the larger care flows of geriatric hospital care.

This study will evaluate whether a proactive primary care intervention into a predicted risk population of the elderly results in care that is more effective and of higher quality than that of a control group who receive standard care. In addition, in a set of parallel substudies, factors that may facilitate or act as barriers to the development of healthcare for older persons will be studied from several perspectives, including those of the elderly themselves and of the healthcare of the elderly.

\section{METHOD AND ANALYSIS}

The study consists of two parallel lines of research. The first, linked to the primary scientific question, is an intervention study of proactive care for older persons in primary care. The second is a set of substudies on different perspectives of elderly care, ranging from the patient, the professionals and governance to societal aspects. An overview of the project and timeline is presented in figure 1 .

\section{Patient involvement}

The public was represented by healthcare politicians with responsibility for the healthcare of the elderly. They supervised, participated in the construction of, and approved the aims and the contents of the study. They follow their progress of the project every 6 months. The patient's perspective of the study is obtained by in-depth interviews at different time points of the study.

\section{Intervention study of proactive care for older persons in primary care \\ Primary scientific question}

Can the prediction of frail older individuals at high risk of hospital care, combined with proactive healthcare, lead to a decrease in healthcare use and costs?

\section{Design, randomisation and setting}

This intervention study is designed to follow a shift in the paradigm of elderly care that had already been decided by the care providers. This led us to use a study design that enables us to detect the real-world effectiveness

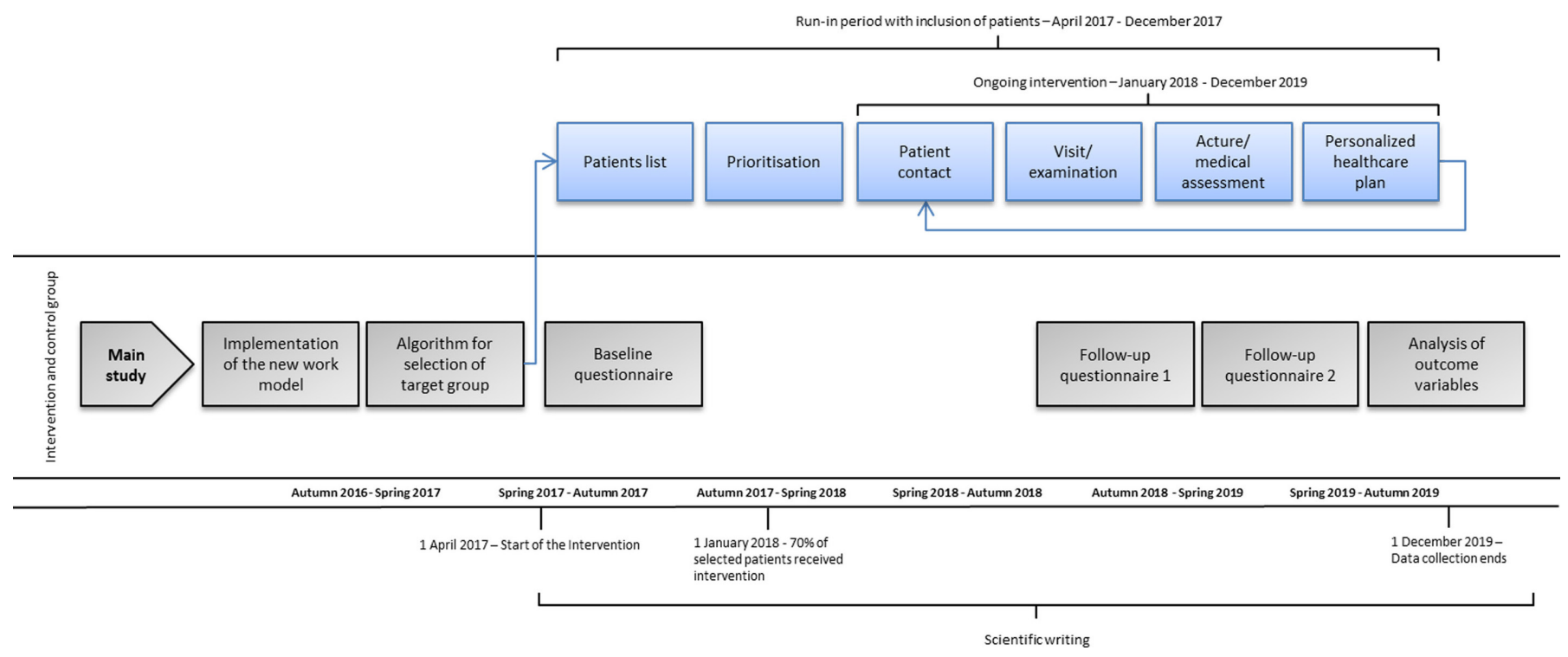

Figure 1 Overview of the project over time. 
of the intervention in a broad patient group in a real, non-selected clinical context with clinically meaningful outcome parameters. Consequently, our design follows a selected pragmatic clinical trial model, in which defined primary care health centres using the new work routines constitute our intervention group, and the remaining centres are used as controls. ${ }^{9}$

The pragmatic clinical trial follows the fact that the intervention to be provided is close to the future modus operandi of healthcare for the elderly but still allows a scientific evaluation before it is implemented further in healthcare organisations. It is a prospective, controlled, multicentre study performed in primary care centres in southeast Sweden. A case-finding algorithm (prediction for hospital care) is used to identify eligible persons within the whole population in the region. The intervention will be performed at nine selected primary care centres (provided by the sponsoring County Council of Östergötland), and the predicted patients there form the intervention group. A similar number of control patients with similar risk scores (for hospitalisation) are predicted in healthcare centres with characteristics similar to those in the intervention centres, but they receive care as usual, and these centres are not made aware of the control patients. There is no randomisation at the patient level, but the case-finding algorithm was used in the selection, and the patients with the highest risk scores were included until the preferred number of patients was reached. There was no randomisation of healthcare centres; these were provided by the healthcare sponsor (County Council of Östergötland). The control healthcare centres were matched in terms of location (city and countryside), size and socioeconomic distribution. A prestudy analysis of the primary outcome measures of the two patient groups revealed no significant differences between them.

\section{Sample size}

A pilot study (not published) showed that $60 \%$ of the target population had at least one hospitalisation during a 12-month period. The hypothesis is that this figure will be reduced by $20 \%$ in the intervention group in this study. A sample size calculation based on this reduction, a power of 0.8 and a significance level of 0.05 led to a minimum of 270 participants per group. Considering the frail and elderly population, we estimate a $40 \%$ drop-out rate, and we have therefore increased the sample size to 378 per group. Since we are using a pragmatic clinical trial design, featuring heterogeneity within both the participating population and the participating healthcare centres, this reduces the likelihood of detecting meaningful changes; therefore, it is reasonable to double the number of participants per group, giving a final number of 800 included individuals per group.

\section{Prediction of patient cases}

The prediction model is described elsewhere (manuscript submitted). In short, the data were obtained between November 2015 and October 2016 from the computerised information system of the County Council of Östergötland, where statistics for all the healthcare in the county is stored. For example, for the whole population, there are records of the number of visits to primary or hospital care, number of days in the hospital and diagnostic codes for each visit. We used an in-ward hospital stay between November 2016 and January 2017 as the dependent variable. The prediction variables are based on a previous study, ${ }^{4}$ including number of general practicioner (GP) visits and International Classification of Diseases, 10th Revision codes, use of assistive technology, emergency room visits, age and gender. The aim is to identify participants aged 75 or older who are likely to be hospitalised during the next 3 months. Risk scores were calculated for all individuals using logistic regression. Individuals were ranked according to the risk scores (for hospital care), from high to low. A cut-off value was chosen so that 800 individuals from the participating healthcare centres with the highest scores were selected for proactive intervention for a period of 2 years. The same cut-off value was then used to choose individuals from the control healthcare centres.

\section{Evaluation form}

A four-page evaluation form has been developed and is used to standardise the evaluation of each individual (the Primary Care Assessment Tool for the Elderly (PASTEL)). The goal is to create a time-efficient, easy-to-use tool for a doctor-nurse team. It is intended to be used by primary care nurses and doctors with different levels of experience. The PASTEL form is based on the holistic approach of Comprehensive Geriatric Assessment, ${ }^{6}$ which can be regarded as a combination of diagnostic and therapeutic processes where problems are identified and managed. The assessments cover medical, psychiatric, functional and social domains required to enable a multifaceted therapeutic plan.

It also includes the Clinical Frailty Scale. ${ }^{10}$

The form contains three parts. The first consists of an interview guide with mostly multiple-choice questions and a self-rating of health. The second part is a checklist for a brief physical examination and laboratory testing, a medication review and questions about the individual's opinion about their present and future needs for care. The third part is used for a team meeting to make a common estimation of frailty and to decide on the need for further investigation and actions to support the elderly person in order to enhance recovery and promote independence.

\section{Intervention}

The intervention group is approached by a primary care team who evaluates the client's social and medical condition and establishes a proactive care plan for individuals in need. The primary care team is represented by the GP responsible for the patient, a registered nurse (RN) dedicated to elderly care and, when needed, a physiotherapist, occupational therapist and/ 


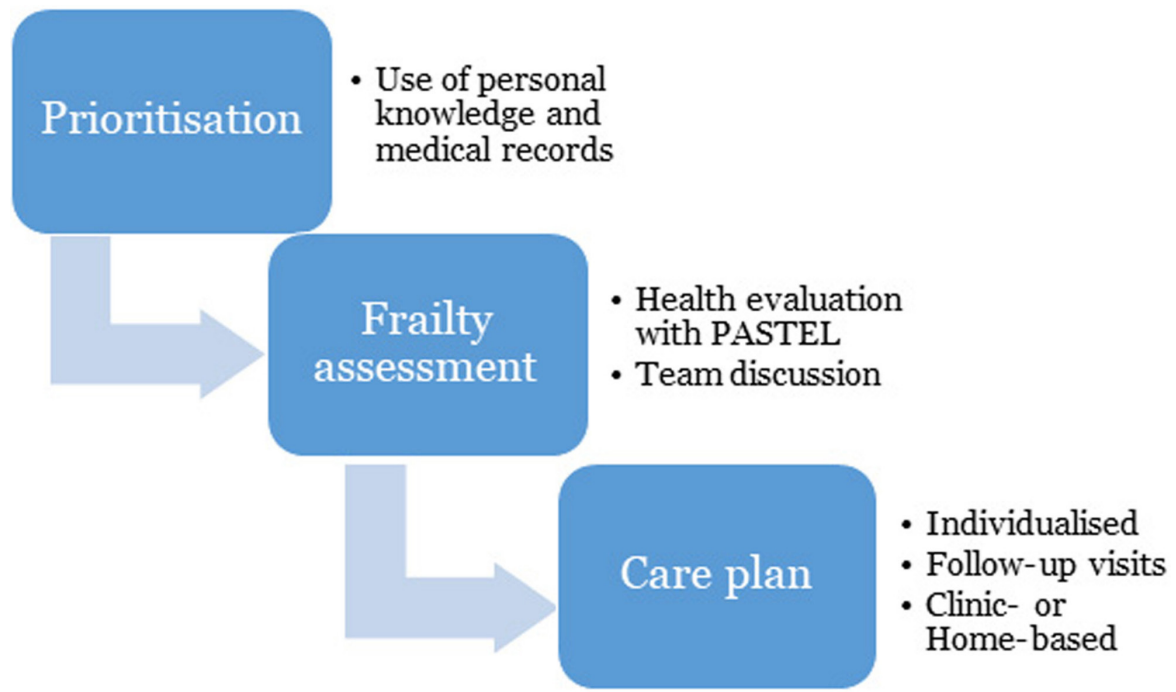

Figure 2 Overview of the intervention. PASTEL, Primary Care Assessment Tool for the Elderly.

or social worker. The proactive intervention consists of a complete check-up/follow-up and intervention into medical, psychiatric, functional and social aspects of the client in a stepwise, resource-differentiated way according to needs based on the clinical judgement of the team (figure 2).

The evaluation process used communication over the phone, as well as visits to the primary care centre, depending on the priority of the client's needs. Examples of common actions/measures are evaluation of medication, initiation of home care, diet counselling, advice on physical activity, and support for loneliness and isolation. The formation of an 'elderly team' with dedicated nurses who function as personal nurses for the frail individuals is the key component of the intervention, together with the standardised evaluation of frailty based on comprehensive geriatric assessment.

\section{Outcome measures for intervention}

Primary outcome measures are healthcare cost, number of hospital care episodes, hospital care days and mortality. Secondary outcome measures are the number of outpatient visits, cost of social care and informal care, number of drugs, number of prescribed drugs not recommended for the elderly, health-related quality of life (HRQoL), cost-effectiveness, sense of security and functional ability.

Data on healthcare consumption will be obtained from the administrative healthcare database, and data on healthcare costs will be obtained from the cost-perpatient database. Costs for social care and informal care are collected from the 'Questionnaire for the patient's perspective and in-depth patient data' (see below) and will be estimated by the number of contacts multiplied by a defined unit cost. Use of medications at the group level will be studied by extracting group data from the National Medication Database before, during and after the study.
Statistical analysis for intervention study

Primary and secondary outcome measures for intervention versus control population will consist of analyses for completed years 1 and 2, respectively, using the intention to treat (ITT) and last observation carried forward (LOCF) method. Differences in means and proportions between groups will be analysed using a t-test. Differences in categorical data will be analysed using a $\chi^{2}$ test. If the baseline mean risk score differs between the intervention and control groups, primary outcomes adjusted for risk score will be analysed using linear or logistic regression.

\section{Monitoring}

Every 6 months, each primary care centre is monitored by the project group, providing opportunities for dialogue and problem solving. Every 6 months, or earlier when needed, each primary care centre reviews the patients included in the study and actions are considered, depending on the results of the review. Every 6 months, the primary care teams gather for a network meeting to discuss common issues and share experiences.

\section{Data management}

No biomaterials are included in the study. All patient data will be processed lawfully according to the General Data Protection Regulation. The data file used for the prediction model, for the intervention group and for the control group was retrieved from Region Östergötland's administrative databases. The data file that will be used for analysis in both the main study and the substudies contains personal data from Region Östergötland's administrative databases, as well as data reported by the patient. The file will only be available to the overall project manager and individuals responsible for each subproject, that is, the coauthors of this paper. The file will be stored in databases with a high level of security at Region Östergötland and Linköping University and will also be protected by personal passwords. Questions regarding data are replied 
to through the corresponding author on request. We used the SPIRIT reporting guidelines. ${ }^{11}$

\section{Substudies on different perspectives of elderly care, from patient to professionals and governance to societal aspects Scientific questions}

What are the experiences of the previous and new healthcare model for older people from a wider individual, social, professional and societal perspective? What are the governance mechanisms that may facilitate or act as barriers to the development of healthcare for older people? What is the cost-effectiveness of the intervention in comparison to care as usual?

Based on these overall questions, there are four main research perspectives with specific research questions:

1. The perspective of the older patients and their families: how does the change in healthcare provision towards proactive primary elderly care impact on individual participation and subjective well-being, as well as objective indicators of quality of life beyond health? How does the change in healthcare provision towards proactive primary elderly care shape the receipt of informal help and support from spouses, offspring and the wider network? These research questions are studied through a questionnaire from the patient's perspective and interviews from the patient's perspective.

2. The professional perspective on the healthcare system: how does the change in healthcare provision towards proactive primary elderly care change the satisfaction and support of the professionals within the healthcare system? The methods used are qualitative studies of selected parts of the healthcare system and implementation studies (see below). An implementation study explores the organisational readiness to implement the new work routines in primary care. Investigating organisational readiness can provide knowledge about early factors that are important for implementation.

3. The governance perspective: what are the mechanisms and explanations for today's elderly care, from the political level down to operative healthcare management? The methods used are implementation studies using semistructured interviews.

4. Cost-effectiveness: what is the cost-effectiveness of the intervention compared with usual care? Data will be collected through questionnaires concerning patient health-related outcomes and from administrative registries of healthcare consumption and costs.

\section{Methods for substudies}

\section{Questionnaire for the patient's perspective and in-depth patient}

data

We will study how the change in healthcare provision impacts on individual well-being, the support they receive from their private networks of families and friends, and their satisfaction with and the support of the healthcare system. A longitudinal study design enables us to follow changes over time. Moreover, we will analyse whether the focusing of care contributes to the life-course accumulation of (dis)advantage in old age and how this contributes to social inequality dynamics. The longitudinal patient questionnaire study collects data on three occasions over a period of 36 months: baseline before intervention, after completed years 1 and 2 , respectively. The information from the questionnaires will be combined with a registry-based assessment of social-structure and life-course information at baseline and referenced with nationally representative life-course data on health, occupation and family from Statistics Sweden, and the National Board of Health and Welfare (Socialstyrelsen). Measures in the questionnaire include the EuroQol-5 Dimensions-3 Levels (EQ-5D-3L) and EuroQol-Visual Analogue Scale (EQ-VAS) ${ }^{12}$ for HRQoL estimates, activities of daily living/functions by the ADL Staircase ${ }^{13}$ and RAND-36 for self-reported functional health. ${ }^{14} \mathrm{~A}$ measure for sense of security in care is also used (Sense of security in-care patients, SECP). ${ }^{15}$ The dizziness handicap inventory is used to detect the presence of a risk of falls ${ }^{16}$ and its related health consequences. A visual analogue scale $(0-100 \mathrm{~mm})$ and a pain-drawing instrument is used to evaluate pain experience. ${ }^{17}$

\section{Interviews from the patient's perspective}

In one subproject, we focus on how frail older people experience care services if included in the intervention. Twenty semistructured interviews with elderly patients will be conducted. A selection of elderly patients will be made, and this selection will include patients who have experiences of the intervention. Interviews are intended to provide access to the feelings, thoughts and experiences of patients. The starting point is that the interview is a knowledge-producing activity, and it is during the interview and in the interaction with the individual and the researcher that knowledge is produced. ${ }^{18}$ Another subproject aims to investigate how the elderly experience their everyday lives and the opportunities for rehabilitation from an availability and participation perspective. Do the elderly receive the rehabilitation they consider themselves to need? A qualitative study with a strategic selection of approximately 20 participants from the intervention group will be conducted.

\section{Qualitative studies of selected parts of the healthcare system}

A qualitative approach (shadowing) ${ }^{19}$ is used to study the working conditions for nurses in primary care in relation to challenges in their professional responsibility connected to their work. The proposed subproject addresses key issues in order to obtain knowledge about how competence levels and the distribution of tasks match the needs of frail older people living at home. The ongoing development in the field of the care of older people can be studied through the concept of task shifting. ${ }^{20}$ Questions of task shifting are implicit in discussions concerning the relationship between general competence and specialist competence within professional groups or the resource deficit in relation to ageing populations. The main aim is therefore to explore and characterise task shifting 
processes in practices, competencies, responsibilities and roles from the perspective of RNs working within the main project. A second aim is to explore the challenges of handling drugs and the pharmaceutical preparations related to nurse practices in home care and how these challenges are processed.

\section{Implementation studies}

In order to meet the future challenges posed by an ageing population, it is important not only to develop and evaluate new care models but also to ensure that these models are implemented successfully by organisations providing care. Three separate studies investigate the implementation of the new work routines for improved care among the frail elderly.

To study the implementation of the model, the project will use a framework that specifies four types (or domains) of determinants, which function as barriers and/or facilitators for successful implementation. Research in implementation science has established that successful implementation depends on an interplay between these determinants: (1) the effectiveness of the strategies chosen to support the implementation; (2) the characteristics of the new practices (routines and methods) being implemented; (3) beliefs, attitudes and motivations among the front-line implementers and (4) the context of the implementation. The framework will provide a basic structure of interviews which will be carried out with representatives of different levels of the healthcare system: from political leadership and primary care management to practitioners on the front line of primary care. Study I focuses on the role of professionals in implementing the new work routines, including adopting a holistic approach to care. This study also investigates readiness to change at both an individual level (eg, resources and attitudes) and an organisational level (eg, system that supports change).

Study II. Preliminary results from study I indicate that successful collaboration between primary care providers and the municipalities is essential in achieving proactive care and implementing the new work routines. Indeed, most care of the frail elderly occurs outside of a primary care setting. The second study therefore investigates these conditions or collaborations via interviews with managers representing both organisations. A concept mapping approach will be used to identify and quantify the factors affecting implementation. Interviews will be audio-recorded and transcribed verbatim.

Finally, a third study will focus on investigating the governance mechanisms that influence the present situation, as well as the uptake of new models through policy-making and implementation. Despite good intentions and various policies, Swedish elderly care has not undergone any extensive change; thus, the same challenges and development needs are being discussed today as 20 years ago.

For this reason, this subproject will investigate the mechanisms that facilitate or impede evidence-based policy-making and implementation from the political level to the regional level in elderly care. Questions that will be studied are what influences policy-making and implementation at different levels? What are the strategies for policy implementation? How is policy implementation monitored and evaluated at different levels? Three levels of policy-making and implementation will be studied: level 1: politicians and Ministry of Health and Social Affairs; level 2: state agencies and authorities at national level, that is, NBHW (Socialstyrelsen) and the Swedish Association of Local Authorities and Regions; level 3: politicians, executive boards and managers at county council and regional level. Interviews will be audio-recorded and transcribed verbatim.

\section{Cost-effectiveness}

A cost-effectiveness analysis will also be performed. The primary outcome in the analysis is the incremental cost-effectiveness ratio: cost/quality-adjusted life year (QALY). The QALY weights for the analysis will be derived from the EQ-5D-3L, and the QALYs will be calculated by multiplying the QALY weight with time. The analysis will have a societal perspective meaning that all relevant costs will be included in the analysis. Healthcare use and costs will be retrieved from administrative databases. Information on social care and informal care will be retrieved from the questionnaire. The cost-effectiveness analysis will be performed with a short-term perspective (within trial) and also with a lifetime perspective applying health economic decision modelling.

\section{Statistical analysis for substudies}

The outcomes of the substudies are the number of outpatient visits, cost of social and informal care, number of drugs, number of prescribed drugs not recommended for the elderly, HRQoL, cost-effectiveness, sense of security and functional ability. The measures will be analysed for years 1 and 2, respectively, using the ITT and LOCF method. Differences between groups will be analysed using a t-test. We intend to estimate the cost-effectiveness in terms of cost/QALY from a lifetime perspective using simulation models. The QALY weights will be obtained from the EQ-5D-3L.

\section{Time plan}

An overview is presented in figure 1 . The project started with the development of the case-finding algorithm (manuscript in progress) in 2017. Based on this model, the case-finding process was undertaken at the beginning of March 2017. Selected patients were presented to each healthcare centre for the start of the intervention programme in April 2017. A run-in period of AprilDecember 2017 was used, during which healthcare centres were informed about and introduced to the new work model, and patients were subsequently enrolled onto the programme. All selected high-risk patients will have received an initial healthcare and/or social care plan. More than $90 \%$ of the selected patients were included by the end of December 2017. The intervention/follow-up 
period is planned to last for 2 years, until the end of 2019 (figure 1).

An initial questionnaire was sent to all selected patients in the intervention and control healthcare centres during May-June 2017. The questionnaire will also be distributed to enrolled participants in years 2 and 3. Interviews with professionals in participating primary care health centres and communities were performed during June-September 2017. Interviews with elderly participants in order to capture the patient's perspective on the study were performed during December 2017-July 2018. Interviews with elderly participants in order to capture the patient's perspective on rehabilitation will take place during November 2018-February 2019. Interviews with high-level decision-makers and politicians were conducted during January-September 2018.

A first preliminary outcome analysis after 13 months of intervention will be performed in 2019. The intervention and collection of healthcare data ends on 31 December 2019. The analysis of primary and secondary outcome measures starts in 2020. The scientific writing up and participation in academic conferences has already started for some of the subprojects. The writing period for the intervention study begins in spring 2020. Dialogue with owners/stakeholders at a political level takes place every 6 months during the course of the project.

Possible evidence for changes in elderly care across the whole County of Östergötland will be available in autumn 2020, when the implementation process of the new care model can be broadened. Members of the research group are participating in workshops at a national level concerning healthcare development.

\section{Ethics and dissemination}

The ethical review board judged all aspects of the study, including design and safety. By adding an academic study to an ongoing change in the healthcare process for the elderly, we do not per se include or exclude treatment possibilities for individuals or groups of individuals. The study itself is 'inert' within the healthcare system. Therefore, we do not see that the use of aggregated patient data from the healthcare system can be of any harm to the participants. On the contrary, we find strong ethical motives for the study, which is an academic attempt to detect the real-world effectiveness of a politically determined intervention into a large patient group. The patients who responded to the questionnaire did so using an informed consent.

The data will be presented in scientific journals and communicated at scientific meetings during the period 2018-2022. The outcome data of the study will be presented to the healthcare provider (County Council of Östergötland) for a discussion on the evidence relating to future care models for elderly persons. The data will also be used by healthcare managers and decision-makers for the development of future care models.

\section{DISCUSSION}

Clinical trials on complex healthcare processes are rare and difficult to design with adequate scientific quality. On the other hand, delimited clinical trials may also only be valid within an academic setting, and the outcome may be difficult to reproduce in clinical reality. In order to counteract the scientific challenges facing trials in complex clinical settings and processes, the use of a pragmatic clinical trial design is one, or perhaps the only, alternative. ${ }^{9}$ In terms of the primary context of elderly care, this study is intended to find answers to basic scientific questions about the future healthcare of the elderly.

One challenge for the study was to find older patients in need of healthcare, hopefully before escalating needs would develop. Managing thousands of patients during this screening for possible illness may be impossible in healthcare using face-to-face methods. Statistical models for case findings have recently proven valid and are recommended in the clinical healthcare of old persons ${ }^{8}$ so this modus operandi was used in this study.

There is a great need for improved healthcare for the elderly and a simultaneous knowledge gap regarding scientific data on what care models to use in the future. This study aims to fill some of that gap and may hopefully generate some clinically meaningful data that can be used for the future development of healthcare for older persons.

\section{Author affiliations}

${ }^{1}$ Acute Internal Medicine and Geriatrics and Departmentof Clinical and Experimental Medicine, Linköping University, Linköping, Sweden

${ }^{2}$ Family Medicine, Department of Medical and Health Sciences, Linköping University, Linköping, Sweden

${ }^{3}$ Health Care Analysis, Department of Medical and Health Sciences, Linköping University, Linköping, Sweden

${ }^{4}$ Department of Medicine and Health Sciences, Linköping University, Linköping, Sweden

${ }^{5}$ Department of Management and Engineering, Linköping University, Linköping, Sweden

${ }^{6}$ Rehabilitation Medicine, Department of Medicine and Health Sciences, Linköping University, Linköping, Sweden

${ }^{7}$ Department of Social and Welfare Studies, Linköping University, Linköping, Sweden ${ }^{8}$ Ageing and Social Change, Department of Social and Welfare Studies, Linköping University, Linköping, Sweden

${ }^{9}$ Department of Social and Welfare Studies, Faculty of Health Sciences, Norrköping University, Norrköping, Sweden

${ }^{10}$ Clinical Pharmacology, Department of Medicine and Health Sciences, Linköping University, Linköping, Sweden

${ }^{11}$ Physiotherapy, Department of Medicine and Health Sciences, Linköping University, Linköping, Sweden

${ }^{12}$ Research and Development Unit in Region Östergötland, Linköping University, Linköping, Sweden

Contributors All authors have made substantial contributions to the conception or design of the work, or the acquisition, analysis or interpretation of data. They have participated in drafting or revising the work critically for important intellectual content. The gave final approval of the version to be published. JM: principal investigator, design, analysis and scientific writing. MN: clinical project leader, design, clinical site visits, data collection, analysis and scientific writing. YB: design, analysis and scientific writing. H-JD: design and analysis of the prediction model and scientific writing. MMJ and AP: clinicians and scientists responsible for rehabilitation, design of the postal questionnaire, data collection, analysis and scientific writing. JA and L-ÅL: design and health economic analysis, design of the postal questionnaire, data collection, analysis and scientific writing. PD, KT and 
BP: design and analysis of implementation studies, data collection, analysis and scientific writing. AS, A0 and EC: design of interviews, analysis of the patient's perspective and scientific writing. SK and AM-K: design of the postal questionnaire, analysis of well-being, network support, satisfaction and social inequality based on questionnaire and registry data, and scientific writing. IH and AK: design and analysis of professional perspective and scientific writing. JL: analysis and scientific writing. MW: project coordinator and scientific writing. AA: coinvestigator, analysis and scientific writing.

Funding This work was supported by the County Council of Östergötland and Linköping University from the strategic research fund for 'Health Care and Welfare' (Grant number 2016186-14). Contact person is Dean Johan Söderholm, Deans Office, Medical Faculty, Linköping University. This funding source had no role in the design of this study and will not have any role during its execution, analysis, interpretation of the data, or decision to submit results.

Competing interests None declared.

Patient consent for publication Obtained.

Ethics approval This study was subject to ethical evaluation and was approved by the regional ethical review board in Linköping (Dnr 2016/347-31).

Provenance and peer review Not commissioned; externally peer reviewed.

Open access This is an open access article distributed in accordance with the Creative Commons Attribution Non Commercial (CC BY-NC 4.0) license, which permits others to distribute, remix, adapt, build upon this work non-commercially, and license their derivative works on different terms, provided the original work is properly cited, appropriate credit is given, any changes made indicated, and the use is non-commercial. See: http://creativecommons.org/licenses/by-nc/4.0/.

\section{REFERENCES}

1. Banerjee S. Multimorbidity-older adults need health care that can count past one. Lancet 2015;385:587-9.

2. Soong J, Poots AJ, Scott S, et al. Developing and validating a risk prediction model for acute care based on frailty syndromes. BMJ Open 2015:5:e008457.

3. Collerton J, Davies K, Jagger C, et al. Health and disease in 85 year olds: baseline findings from the Newcastle $85+$ cohort study. BMJ 2009;339:b4904.

4. Nägga K, Dong HJ, Marcusson J, et al. Health-related factors associated with hospitalization for old people: comparisons of elderly aged 85 in a population cohort study. Arch Gerontol Geriatr 2012:54:391-7.

5. Edmans J, Bradshaw L, Franklin M, et al. Specialist geriatric medical assessment for patients discharged from hospital acute assessment units: randomised controlled trial. BMJ 2013;347:f5874.

6. Ellis G, Whitehead MA, Robinson D, et al. Comprehensive geriatric assessment for older adults admitted to hospital: meta-analysis of randomised controlled trials. BMJ 2011;343:d6553.

7. Panza F, Lozupone M, Solfrizzi V, et al. Different Cognitive Frailty Models and Health- and Cognitive-related Outcomes in Older Age: From Epidemiology to Prevention. J Alzheimers Dis 2018;62:993-1012.

8. NICE. National Institute for Clinical Excellence. Multimorbidity: clinical assessment and management (NICE clinical guideline 56), 2016

9. Ford I, Norrie J. Pragmatic Trials. N Engl J Med Overseas Ed 2016;375:454-63.

10. Rockwood K, Song X, MacKnight C, et al. A global clinical measure of fitness and frailty in elderly people. CMAJ 2005;173:489-95.

11. Chan AW, Tetzlaff JM, Altman DG, et al. SPIRIT 2013 statement: defining standard protocol items for clinical trials. Ann Intern Med 2013;158:200-7.

12. EuroQol G; EuroQol Group. EuroQol-a new facility for the measurement of health-related quality of life. Health Policy 1990;16:199-208.

13. Sonn $\mathrm{U}$, Asberg $\mathrm{KH}$. Assessment of activities of daily living in the elderly. A study of a population of 76-year-olds in Gothenburg, Sweden. Scand J Rehabil Med 1991;23:193-202.

14. Orwelius L, Nilsson M, Nilsson E, et al. The Swedish RAND-36 Health Survey - reliability and responsiveness assessed in patient populations using Svensson's method for paired ordinal data. $J$ Patient Rep Outcomes 2017;2:4.

15. Krevers B, Milberg A. The instrument 'sense of security in care-patients' evaluation': its development and presentation. Psychooncology 2014;23:914-20.

16. Jacobson GP, Calder JH. A screening version of the Dizziness Handicap Inventory (DHI-S). Am J Otol 1998;19:804-8.

17. Margolis RB, Tait RC, Krause SJ. A rating system for use with patient pain drawings. Pain 1986;24:57-65.

18. Patton MQ. Qualitative research \& evaluation methods. London: SAGE, 2002

19. McDonald S. Studying actions in context: a qualitative shadowing method for organizational research. Qual Res 2005;5:455-73.

20. WHO. Task shifting: Rational redistribution of tasks among health workforce teams: Global recommendations and guidelines. Geneva: World Health Organization, 2008. 